\title{
Media Noise: the problem of hygienic rationing and personal well-being
}

\author{
Alla Polyanina ${ }^{1,2, *}$ \\ ${ }^{1}$ Lobachevsky University, Gagarin Avenue 23, 603087 Nizhny Novgorod, Russia \\ ${ }^{2}$ Samara State University of Railway Transport, Komsomolskaya Square 3, 603087 Nizhny \\ Novgorod, Russia
}

\begin{abstract}
The article raises the problem of hygienic rationing of a special type of noise, the nature of which stems from the sources of public information - the mass media. The author develops a new concept of comprehending this type of noise - the concept of Media Noise. Such noise is associated with the special nature of its perception and consumption in the background mode that acts as a risk factor for human health and well-being. The author points out such specific characteristics of the media noise that relate it to certain risk factors, namely: hyperstimulation of the auditory analyzer and the inability to control its implementation (work), that is, the compulsion of perception. This second key feature of the media noise is similar to mental violence, if the audio signal is used by an external subject, for example, in public places or transport, and its broadcasting excludes the possibility for the perceiving subject to influence upon the source of noise (a screen or a player).
\end{abstract}

\section{Approaches to Conceptualization of Information Hygiene}

Hygienic rationing is aimed at improving the environment, including preventing the pressure of information influence on the human body. Initially, the problem of adverse information influence was considered in the aspect of intellectual work as professional hygiene in 1871 and was widely developed in the same area [1]. New problems for human well-being, provoked by unprecedented technological and technical progress, are related to the increasing strength of the information environment factors, in particular the flow of information. Conceptualization of this problem caused domestic researchers to identify in 1995 a subdiscipline of hygienic science and the justification of its object [2]. It was proposed to interprete information hygiene as "a subdiscipline of medical science that studies the laws of the influence of information on the mental, physical, and social wellbeing of a person, his/her ability to work, life expectancy, public health of society, developing standards and measures to improve the information environment and optimize intellectual activity" [3]. Information hygiene scientists consider information overload as a cause of the avalanche of mental and somatic information-dependent diseases. These consequences are particularly dangerous for certain social groups, for example, the most vulnerable one - our children.

${ }^{*}$ Corresponding author: Alker@yandex.ru 
Prevention of negative effects on human health and well-being requires standardization and rationing of the functioning of physical quantities that can be measured, namely, the action and parameters of the information signal. Within the framework of information hygiene, hygienic rationing of signals - carriers of visual and auditory information, as well as the limits of information loads, became a subject of R\&D. Thus, the Russian sanitary legislation defines the term of "dosing" in relation to certain indicators, for example, the level of sound, light, the number of read and entered signs. The researchers point to the insufficiency of hygienic quantitative and qualitative rationing in relation to: determining the reference standards for distinguishing (the necessary "gap") sound - the information carrier and noise - the combination of sounds, as well as distinguishing between the object and the background ("contrast of the object with the background"); reference standards for the speed of information flow; reference standards for the complexity of information, including the semantics and context of verbal information, as well as the complexity of the dynamics and environment of movement of the image (figure) [4].

Information hygiene is aimed at preventing the negative impact of information on a person and social groups, which is expressed in various forms of distress and disorders of social well-being, such as somatic health disorders: psychoemotional overstrain and its consequences (diseases of the cardiovascular, digestive system (peptic ulcer), immune systems of the body, cancer; mental disorders, anxiety, depressive and panic disorders, new types of phobias, such as nomophobia (fear of being left without a mobile phone), Internetdependent suicides, behavioral disorders, additive behavior, including a pathological attraction to gambling (ludomania), and addiction to social media. There are also other negative consequences of information influences of both individual psychological and general social aspects: "mosaic thinking" - a superficial perception of information, the destruction of spatial-temporal and causal relationships and a qualitative change in the complexity of thinking: from linear to nonlinear [5]; blurring of self-identification, "extended self-identification", when the identity of a modern person acquires the properties of hypertext; dysfunction of the volition - "paralysis of decision-making"[6]; stereotyping of consciousness [7]; the phenomenon of phantom vibration or mobile phone ringing [8] and much more.

Prevention tools are quantitative and qualitative hygienic rationing of production, distribution, consumption, storage, and reproduction of information, as well as measures to enhance the organization and recuperation of the information space.

\section{Methodology}

Using the latest scientific developments and the requirements of sanitary legislation, we attempt to describe the concept of a particular type of risks associated with the non-targeted consumption of an information signal, "updated and maintained with the help of technology"[9], viz. the media noise.

Currently, hygienic rationing describes such physical factors as sound pressure, sound level and its acceptable exposure limits (AEL), exceeding of which can lead to significant anxiety and major changes in the state of noise-sensitive systems and analyzers. Cassification of noises that affects a person is also of some interest, for example, by time characteristics: constant and non-constant (fluctuating, intermittent, and pulsed) noise.

However, the power of the pressure of the acoustic signal on the receiving system (the auditory analyzer) is measured in units that cannot be fully called "a unit of measurement, at least in the sense of, for example, volts, meters, grams, etc."[10]. Decibels is a ratio of values that requires a reference value, a reference point, for the possibility of universal application, since the acuity of sound perception may differ from one person to another, and loudness is only "a judgment about the intensity of sound made by a person on the basis of 
auditory sensation". Hygienic rationing is based on the measurability of environmental factors as a principle of regulation. Therefore, despite the conventionality of the reference value of the perception of sound, wave motion in any medium, a unit of measurement is required.

In the selected research field, we are interested in the principles of hygienic rationing, as well as the initial principles that interpret the factors of information impact on human wellbeing. The levels of epidemiological risks are determined in accordance with the dosebased principle of exposure rationing. The identification of acceptable levels of harmful environmental factors, namely the sound level, is based on the prediction of the effects of exposure on the body using the "dose-effect" ratio through quantitative characteristics of the intensity and duration of exposure. However, the measurement of the effects of the impact of non-targeted consumption of an information signal, "updated and maintained with the help of technology", that is, a signal created and transmitted by the media, needs to take into account the specific features of information encoding in the brain, identifying the consequences of processing, perception, and memorization of information. These features can be recorded using neurophysiological methods (emotional stress, information overload: fatigue, tiredness), diagnoses (manias, addictions, phobias, etc.) and socio-psychological questionnaires. Unlike visual information, the perception of sound coincides with the moment of its realization (its reality), so it is impossible, when evaluating the possibilities of controlling one's own perception, to use an analogy with the possibility of individual reduction in intensity (or blurring) of visual information, for example, when using sunglasses or focusing the gaze (control of the perception organs). It is not possible to independently establish a barrier to the penetration of auditory information without restrictions imposed on spatial orientation systems, as is the case with the use of headphones or other means of protection, such as earplugs. Unlike a number of other physical factors, noise is always perceived by the body as an irritant. At the same time, the analyzer of incoming auditory information is not adjusted to adapt to noise.

The researchers determined that there is a difference in people's sensitivity to noise. Thus, "in natural and laboratory conditions, it was determined that for insensitive individuals, the current level of aircraft noise is $95 \mathrm{dBA}$, for normally sensitive individuals $-85 \mathrm{dBA}$, and for hypersensitive individuals - $65 \mathrm{dBA}$ " [11]. In 2004, the specific effect of noise on the hearing organ was studied using tonal audiometry and polling. The research methods made it possible to take into account the noises created by "playing equipment in apartments, cars, on the streets, noises from water and sewer equipment, and elevators in buildings, as well as noises from television and radio broadcasts, anti-theft devices, and sounds made by people and animals", excluding transport noises. Such a comprehensive accounting of noise sources allowed the authors of the study to develop new units of measurement "Silence Index" (ST, \%) and "Noise Load Factor" (NLF, \%) and calculate them. The authors note that the increase in the Noise Load Factor up to $50 \%$ or more indicates an extremely high level of sound load on the human hearing organ. As a result of the performed audiometry, it was determined that people residing in noisy places and individuals exposed to high levels of noise load, begin to experience age-related hearing loss (presbiocusis): men - at the age of 25 years old, and women - at the age of 30 years old, which is 5-7 years earlier than in people living in quiet places and individuals exposed to a normal levels of sound load (NLF in the range between $25-40 \%$ ). Another study by the same authors determined the dependence of people's assessment of test tasks on the noise load of places of residence. If those residing "in quiet areas, assessed the usual mental load as a light test task, then the majority of those living in very noisy areas, assessed this standard mental load as a heavy test task, i.e., this load was assessed inadequately" [12]. 


\section{Results}

Noise is a complex of sounds, a complex of wave motions, and is recognized as one of the epidemiological risks. However, the risk factors (in terms of hygiene) are only indicators of its intensity, most often loudness, with the exception of the above study of silence, which can be called a pilot in this sense. But we are not so much interested in the volume of sound as in other parameters of acoustic information that affect the well-being of a person. These are: the concentration of noise (the number of simultaneously realized sounds, different sources); the complexity expressed in content fragmentation (semantic discrepancy); the compulsive nature of its presentation, and the compulsion of a corresponding reaction (hyperstimulation). At the same time, if noise sources are only technical sources of information scaling, viz. playback equipment, screens, loudspeakers, etc. and not natural, man-made or domestic ones, such noise is defined by us as media noise.

Information noise is close in meaning to media noise and already has some scientific description. The well-known noise model of communication developed by C. Shannon $\mathrm{W}$. Weaver describes information noise as interference that generates the background for communication and leads to a distortion of the message and of the recipient's perception of the message [13]. Information noise is associated with elements of the formal organization of the text, which "hinder the perception of information, distort it or make it impossible to receive it" [14]. In a broad sense, information noise is defined as "any causes and factors that make it difficult to perceive the transmitted information" [15], that is, as "random lowquality information" or "information redundancy in the communication environment that causes a functional disorder of its systems" [16]. Attempts have been made to draw an analogy with acoustic noise and to focus on such noise characteristics as the strength and frequency of interference [17]. Some authors believe that information noise also occurs due to the excessive accumulation of necessary (relevant), but repetitive information [18]. There is also such a quality of information noise as "multilayering", redundancy of information that "does not rely on the original data" [19]. Therefore, information noise is defined through such categories as: interference, environmental factors, and media text organization.

The researchers' understanding of information noise is focused on such properties of the information flow as the irrelevance of its content, the difficulty (or impossibility) of filtering and controlling it, as well as the high concentration (intensity) of the flow. Information noise is characterized by unconsciousness on the part of the consuming subject. The concept of information noise is associated with the phenomenon of information flows characterized by a certain source, direction, intensity, speed, frequency, degree of constancy, structure, volume and density, type of media, information capacity, and degree of use.

According to certain researchers, the media noise resides within a certain media channel, which can always be identified [20]. Media noise is considered by us, not from the standpoint of the Shannon approach as interference in communication, but as an artificial stimulation of the organs of perception by means of a media signal (for example, radio in a car or store, advertising, and background TV viewing). In accordance with this approach, media noise incorporates signals from any sources of mass information, including advertising. Using McLuhan's expression about the "expanding" effect of interaction with the media, the media noise is represented as a factor of forcing the individual to artificially "expand" the organs of reception, to go beyond their physiological limits.

The proposed understanding of the media noise is focused not so much on the semantic (notional) aspect, but rather on the problem of threshold exploitation of the perceptual organs and their hyperstimulation, as well as on the coercive nature of the auditory signal and the corresponding social effects. Thus, hyperstimulation of the reception organs is 
associated with such a feature of the media noise as the density and concentration of media signal flows, as well as the level and strength of sound; the coercive nature of the media noise is associated with the signal source (screen, player, etc.) which cannot be controlled by the perceiving subject. This latter characteristic of the media noise is actualized in a special form of media consumption - the background one, when the media acts as a background for other activities. The very perception of a semantically rich (including emotionalized) information signal is not targeted and is often not realized. At the same time, control over the source (device) transmitting the signal is either outside the control zone of the subject or is not perceived by him as significant or necessary to prevent negative consequences, since the signal is recognized by the subject as not requiring awareness and "involvement".

In a large number of studies of various aspects of media consumption, there are no special studies aimed at researching the nature and consequences of background consumption of media information. Only some sources analyze the background consumption, but even then it is done with the only purpose of assessing the efficiency of advertising embedded in the media, the so-called native advertising. The weak research interest towards the problem of background media consumption is explained by the absence of its obvious negative effects, as well as the generally recognized "harmlessness" of the broadcasting device during other activities, such as driving a car, walking, or doing household chores. Listening to an audio signal is considered, if not desirable, then harmless in many situations and locations. The operation of the audio signal sources, screens or players, is not considered to be forcing the auditory analyzer to react and to become a subject of hyperexploitation. However, the generation of a special social pattern of media consumption (and communication) observed by researchers indicates the standardization of human interaction with online information resources and the emergence of patterns of consolidation of background media consumption at the generational level as a desire to bring the surrounding information space to the "standards" of the familiar (and, therefore, comfortable) or as maintaining a position in a relevant group through constant consumption of the media signal [21].

Non-targeted consumption of the media signal in the background mode, that is, in the "main tone, general space" mode, can be aimed at constructing a certain social situation ("entertainment", "home", "market", "travel", etc. ) and contributes to the allocation of the "main elements" of the situation in the form of corresponding psychological states, for example, "I'm home", "I'm traveling", "Busy morning", etc. This explains the demand for such a routine, which, as it were, creates a space for a specific social practice, "activates" its action, that is, affects its components and subjects in this situation, as a natural fragment of social life. However, the compulsive nature of the media noise and the lack of reliable scientific data on the possible negative consequences of continuous operation of the auditory analyzer refer it to risky phenomena.

Since the most popular media product consumed in the background mode is music, it is interesting to analyze the effects of listening to it. Many authors note this property as a positive reinforcement, that is, pleasure. It has been suggested that the reinforcing aspects of music are caused by shifts in the level of emotional activation [22]. The emotional response to music is always accompanied by the response of the autonomic nervous system [23], the cardiovascular system [24], and the hormonal sphere. The latter modulates the activity of the immune system [25].

Foreign researchers in 2019 found a significant deterioration in the ability of people to perform tasks and a distortion of labor productivity and creativity when listening to background music, regardless of the presence of semantic content (song lyrics) and the direction of emotions. At the same time, it turned out that quiet library noise has no effect, since, as the researchers believe, it is a "stable environment that is not so destructive" [26]. 
The difference in the influence of music and "library noise" on a person from the point of view of the proposed concept of media noise can be explained by the difference in the origin and nature of these noises. Music noise refers to media noise, and library noise refers to natural or man-made noise. This confirms the danger of background noise, the source of which belongs to the media sphere.

The effectiveness of problem solving, work and creativity is related to the function of attention, and the influence of noise is related to the problem of its distraction. The problem of attention allocation and focusing on two or more stimuli has been studied for more than a century. As early experiments showed when performing two types of activities simultaneously, one of them requires complete automation [27]. Distraction of attention an involuntary movement of attention-occurs when the action of extraneous stimuli that are not related to the activity being performed. Distraction as a result of the influence of the media is external, that is, it occurs as a result of external, and not internal (experiences) stimuli.

Can background music listening be classified as an automated activity that does not require involvement? The answer to this question refers us to emotion as the substantial beginning of the perception of music. Since the perception of music, as noted above, is always accompanied by emotional activation, listening to it in the background mode cannot be considered automated, that is, not having any consequences in consciousness and not requiring orientation and significant mental resources. Therefore, if we recognize the impossibility of high-quality performance of two non-automated activities, background media consumption not only worsens productivity, but also consumes mental resources.

The connection of consciousness with the world through emotion, that is, throught the evaluation of facts, phenomena, and events of reality, is activated when listening to music, due to the so-called ideatory nature of emotions, viz. the ability to be formed in relation to expected and imagined situations, or even to ideas about experienced situations [28]. This is confirmed by the theory of information stress developed by V. A. Bodrov, in particular, the impossibility of arbitrary control of the "emotional processing of information", which does not follow the formal rules of logic [29]. The high speed and intensity of the impact of emotions on the energy state of a person involves information processing resources, the potential of which is limited. And the inability to control the "launch" of the process of emotional response makes the consumption of media, especially in the form of listening to music, not just highly demanding, but imposed, forced, in other words, similar to mental violence. An audio signal that cannot be controlled by the subject increases the requirements of the information environment and the gap between the requirements of the environment and the resources of the body, which raises the problem of the ecology of the information space. Media streams in the background mode automatically "turn on" the body's response, mobilize the mental load that is inadequate to what a person really needs, and force us to process an emotionally rich media signal. Thus, the media noise forces us to experience emotions and, due to the limited resources of the body, leads to information stress.

\section{Summary}

Media noise is an environmental factor where the signals of various media sources are in the background and not controlled by the perceiving subject, force all sensory systems (organs of perception) to react, and to interact with the media source, automatically mobilizing the energy systems of the body, that is, a factor that contributes to the development of information (emotional) stress.

Media noise correlates with information stress as a cause and effect [30]. Media noise may cause information overload ("media overload") and stress. The probability of negative 
consequences depends on objective and subjective factors, which we call the parameters of media noisiness. Media noisiness is defined as the saturation of a person's living space with media noise, and is determined by calculating the frequency and duration of stay under the media streams, the ability of the subject to establish control over the source of information, and the characteristics of the situation of forced background media consumption. The need for scientific understanding followed by hygienic rationing of the media community is defined by the scale of digitalization of modern society and the total mediatization of human life.

\section{References}

1. F. F. Erisman, A Short Textbook on Hygiene (1898)

2. A. L. Eryomin, Theses of the I-st International Conference "Ecology and Development of the North-West", 238 (1995)

3. A. L. Eryomin, International Journal of Environmental Studies, 54, 241 (1998)

4. A. L Eryomin, Hygiene and Sanitation, 4, 351 (2020)

5. K. G. Frumkin, Ineternum, 1, 26 (2010)

6. J. Doomen, Journal of Information Ethics, 18, 27 (2009)

7. T. Chamorro-Premuzic, The Guardian, 13 (2014)

8. D. J. Laramie, ProQuest. Alliant International University, 136 (2007)

9. D. Petrenko, Philosophy and Social Sciences, 2, 10 (2015)

10. R. Taylor, Penguin, 275 (1975)

11. O. O. Nekipelova, M. N. Nekipelov, T. I. Shishelova, Modern High Technologies, 2, 27 (2005)

12. O. O. Nekipelova, M. I. Nekipelov, T. I. Shishelova, E. S. Maslova, Modern High Technologies, 9, 115 (2005)

13. C. E. Shannon, A Mathematical Theory of Communication (1948)

14. A. V. Kuznetsova, Problems of Information and Entropy in Media Text (2012)

15. S. P. Myasoedov, Personnel Management, 3, 55 (2005)

16. V. P. Poludina, The Journal of Sociology and Social Anthropology, 5, 386 (2011)

17. V. P. Galkin, Problems of Scientific and Extra-Scientific Cognition, 5, 59 (2005)

18. A. D. Ursul, Information Noise (2009)

19. N. V. Yavdolyuk, Sovietnik, 6, 1 (2011)

20. N. V. Kobrin, Philological Sciences. Questions of Theory and Practice, 7, 86 (2014)

21. D. F. Mironov, Bulletin of the St. Petersburg State University of Culture and Arts, 4, 24 (2015)

22. G. S. Radchenko, Features of EEG indicators and vegetative regulation under the influence of musical fragments with different tonal modulation (2017)

23. R. J. Ellis, J. F. Thayer, Music Percept, 27, 317 (2010)

24. H. J. Trappe, Heart, 23, 1868 (2010)

25. S. Koelsch, W. A. Siebel, Trends Cogn. Sci., 12, 578 (2005)

26. E. Threadgold, J. E. Marsh, N. McLatchie, L. J. Ball, Applied Cognitive Psychology, 33, 874 (2019)

27. V. Wundt, Fundamentals of Physiological Psychology (1908) 
28. A. N. Leontiev, Needs, Motives and Emotions (1971)

29. V. A. Bodrov, Information Stress (2000)

30. A. K. Polianina, Tomsk State University Journal, 57, 215 (2020) 